\title{
Obesity - should we revise indications for treatment with metformin?
}

\author{
Magdalena Kujawska-Luczak¹, Hanna Stankowiak-Kulpa², Ewelina Swora-Cwynar², Katarzyna Musialik ${ }^{3}$, \\ Paweł Bogdański ${ }^{1}$, Joanna Suliburska ${ }^{4}$, Marian Grzymisławski
}

${ }^{1}$ Department of Internal Medicine, Metabolic Disorders, and Hypertension, University of Medical Sciences, Poznań, Poland

${ }^{2}$ Department of Internal and Metabolic Diseases, and Dietetics, University of Medical Sciences, Poznań, Poland

${ }^{3}$ Department of Hypertension and Metabolic Disorders, Clinical Hospital No 1, Poznań, Poland

${ }^{4}$ Department of Human Nutrition and Hygiene, Poznań University of Life Sciences, Poznań, Poland

\begin{abstract}
Introduction: Treatment of obesity with life style modifications often fails; therefore pharmacological treatment has become a very popular approach. Metformin is one of the examined possibilities. The aim of this study was to verify indications for metformin use in obese women based on metabolic and anthropometric parameters assessed by dual-X-ray absorptiometry (DXA), to establish the degree of insulin resistance and its correlations.

Material and methods: Anthropometry, fat measurement by bioimpedance and metabolic profile, including lipids, and oral glucose tolerance test (OGTT) with insulin ( 0 and $120 \mathrm{~min}$ ) were performed in 50 female patients diagnosed with simple obesity, aged 18-40 years. Homeostatic model assessment HOMA-R was calculated for insulin resistance, and area under the curve (AUC) for insulin response. Total, android and gynoid fat distribution, and their ratio $(\mathrm{A} / \mathrm{G})$, were measured by $\mathrm{DXA}$.

Results: From 50 women who entered the study, 33 were classified as insulin resistant (IR subgroup) and 17 as non-insulin resistant (non-IR subgroup), according to their HOMA-R indices. IR women presented higher waist circumference and DXA A/G ratio. The IR subgroup demonstrated elevated fasting triglycerides and glucose (but in the normal range) and a higher insulin response in OGTT (4.1-fold vs 2.5 -fold). From different insulin measurements HOMA index turned out to have the strongest correlations with the metabolic parameters triglycerides and glucose. We found significant positive correlations between android fat and insulin: waist circumference and HOMA-R, WHR and HOMA-R, android fat and HOMA-R, A/G ratio and insulin after OGTT, and $A / G$ ratio and HOMA-R. We found a strong correlation between WHR and $A / R$ ratio.

Conclusions: $67 \%$ of premenopausal obese women were insulin resistant. Measures of DXA visceral fat determined by android fat percentage and android/gynoid ratio were the strongest determinants of insulin resistance. Waist-to-hip ratio might be a simple method for determining the indications for metformin treatment.

Key words: visceral obesity, metformin, oral glucose tolerance test, insulin resistance, dual-energy X-ray absorptiometry.
\end{abstract}

\section{Introduction}

Obesity has become one of the major health problems in many countries worldwide [1]. Insulin resistance is a commonly recognized pathology associated with obesity [2]. Both insulin resistance and visceral obesity play an important role in the pathogenesis of diabetes mellitus type 2 , hypertension, dyslipidaemia and atherosclerosis, which are well-described components of metabolic syndrome [3]. It has been observed that high-fat diets lead to increased glucose, insulin, and triglyceride levels in an animal model and human studies and promote insulin resistance and inflammation [4]. Treatment of obesity with life style modifications often fails; therefore pharmacological treatment has become a very popular approach.
Metformin is one of the examined possibilities. Its preparations reveal many positive actions: they decreas glucose output in the liver and in the gastrointestinal tract, increase peripheral glucose utilization without an increase in insulin plasma levels, and in muscles increase translocation into the plasma membrane of some glucose transporter isoforms. Metformin improves beta cell function, thus improving fasting blood glucose, glycohemoglobin and fructosamine levels. In addition, it decreases triglycerides, LDL cholesterol, plasminogen activator inhibitor-1 and urine microalbumin levels [5]. Nowadays because of its unique action metformin has become widely recommended not only in diabetes, but also in a rising number of metabolic disorders and the prediabetic state. For example, according to the clinical guidelines from the Polish Diabetes Society 2013, 
metformin treatment should be considered in patients at high risk of type 2 diabetes, especially those with co-existing impaired glucose tolerance (IGT) and impaired fasting glycemia (IFG) [6]. Similarly, experts from the American Diabetes Association in 2013 stated that metformin therapy for prevention of type 2 diabetes may be considered for those with IGT, IFG, or with a glycated hemoglobin $A_{1 C}$ of 5.7-6.4\%, especially for those with $\mathrm{BMI}>35 \mathrm{~kg} / \mathrm{m}^{2}$, aged $<60$ years, and women with prior gestational diabetes [7].

Many physicians consider using metformin in obesity treatment. Obesity is considered as a disease when it is accompanied with increased fatness; therefore both high BMI and high fat content are indicators of obesity $[8,9]$. Consequently, in some countries preparations of metformin may be prescribed too often, as the decision of its introduction may be based only on BMI calculation, not a measure of body fat content. Conversely, in other clinical situations metformin may be prescribed too rarely, particularly in those individuals whose high android fat content is in disproportion to low abnormal BMI $\left(30-35 \mathrm{~kg} / \mathrm{m}^{2}\right)$, waist circumference and normal biochemical parameters. Therefore prior to a therapeutic decision in obesity, not only standard anthropometric measures (waist circumference, BMI) and biochemical tests (glucose and lipid fraction concentrations), but also additional measurements - android fat content and the degree of insulin resistance - should be considered as part of the standard approach. Although the newest standards of the European Association for the Study of Diabetes from 2008 did not recommend metformin treatment in obesity [10], some obese patients would gain a therapeutic effect from its pharmacological action. If indications were recognized correctly, metformin treatment would prevent this group of obese from development of overt diabetes mellitus type 2, as it is linked to the degree of visceral (android) obesity and insulin resistance.

Although waist circumference is used as a good predictor of visceral (android) fat content, dual X-ray absorptiometry (DXA) is considered a more relevant method. DXA is a very potent method assessing not only total body fat and percentage of body fat mass, but also other characteristics. Therefore it may a useful tool in prediction of cardiovascular risk in patients with metabolic disorders including obesity.

Various methods can also be used for insulin resistance detection, and although the insulin clamp is a gold standard [11] in clinical settings an oral glucose tolerance test (OGTT) is more frequently carried out [12]. Both glucose and insulin responses two hours post oral stimulation with 75 grams of glucose are important for identification of the type and the degree of abnormalities of the carbohydrate metabolism. The homeostasis model assessment for insulin resistance (HOMA-R) although often used as an indicator of in- sulin resistance, is calculated based on fasting glucose and insulin concentrations and does not inform how high an increase in insulin concentration is noted in an individual post meal. HOMA-R is rather an equivalent to fasting serum insulin as indicated by their strong correlation [13]; therefore the evaluation of post glucose load insulin concentration should be tested by OGTT.

The study was designed in order to verify indications for metformin use in obese, non-diabetic women based on biochemical and anthropometric parameters including android fat content assessed by DXA. We also wanted to establish the degree of insulin resistance in obese female subjects and to detect possible correlations between the degree of insulin resistance and selected anthropometric and biochemical parameters. We aimed to assess the presence of disproportion between waist circumference, BMI calculation and the percentage of visceral fat in obese patients assessed by DXA.

\section{Material and methods \\ Participants}

Female patients $(n=50)$ diagnosed with obesity $\left(\mathrm{BMI}>30 \mathrm{~kg} / \mathrm{m}^{2}\right)$, aged $18-40$, were eligible for the study performed in the Department of Internal Medicine, Metabolism and Dietetics, Poznan University of Medical Sciences and in the Department of Internal Medicine, Metabolic Disorders and Hypertension, Poznan University of Medical Sciences. The exclusion criteria were confirmed diabetes mellitus type 2 , untreated hypothyroidism of any cause, hypertension (BP > 140/90), chronic renal failure (GFR $<60 \mathrm{ml} / \mathrm{min} / 1.73 \mathrm{~m}^{2}$ ), acute or chronic inflammation, smoking, pregnancy, breast feeding and menopause.

The Human Subjects Oversight Committee at the Poznan University of Medical Sciences approved the study (no. 688/09). Informed consent was obtained from each patient eligible for the study.

\section{Anthropometric measures}

Anthropometric measures of subjects wearing light clothing and no shoes were carried out. Weight was measured to the nearest $0.1 \mathrm{~kg}$ and height was measured to the nearest $0.1 \mathrm{~cm}$. Body mass index was calculated as weight in $\mathrm{kg}$ divided by height in square meters $\left(\mathrm{kg} / \mathrm{m}^{2}\right)$. Obesity was recognized when BMI exceeded $30 \mathrm{~kg} / \mathrm{m}^{2}$. Waist circumference was measured at a level midway between the lower rib margin and the iliac crest with the tape all around the body in the horizontal position at the end of normal expiration. Waist measurement was carried out to the nearest $0.5 \mathrm{~cm}$. Hip circumference was measured at the level of femoral trochanters. Waistto-hip ratio (WHR) was calculated for each patient. 


\section{Laboratory measurements}

Blood glucose, insulin and lipids concentrations were assayed in serum obtained from a venous blood sample. Blood was drawn from the antecubital vein following overnight fasting (10-14 h). Serum levels of glucose (glucose 0), insulin (insulin 0), triglycerides (TG), total cholesterol (TC), low-density lipoproteins (LDL-C), and high-density lipoproteins (HDL-C) were assayed with a Dimension EXL with LM Integrated Chemistry System Analyzer. Moreover, the oral glucose tolerance test (OGTT) with $75 \mathrm{~g}$ of glucose was performed according to the standard procedure. Venous blood was drawn before and 2 hours after oral load with $75 \mathrm{~g}$ of glucose and serum level of glucose (glucose 2 ) and insulin (insulin 2) were assayed in both blood samples. Glucose concentration was assessed by an enzymatic method with hexokinase, glucose HK gen.3 (GLUC.3), and insulin concentration was assessed by electrochemiluminescence immunoassay (Cobas analyzer). Homeostasis model assessment of insulin resistance (HOMA-R) was calculated based on fasting glucose concentration $(\mathrm{mg} / \mathrm{dl})$ and fasting insulin concentration $(\mathrm{mU} / \mathrm{ml})$ using the standard equation: (fasting plasma glucose/fasting plasma insulin)/405. The threshold for positive values was established at $\geq 2.5$ and for negative values at $<2.5$.

\section{Bioimpedance}

Bioimpedance (BIA) measures were performed using an Akern device and software. The BIA method uses current of $9 \mathrm{~V}$ passing through the right upper and lower part of the body while the subject stays in a supine position on a bed, wearing light clothes. Electrodes were placed on the dorsal aspect of the right hand, one at the base of the right middle finger and the other at the level of the right wrist. The other 2 electrodes were placed on the anterior side of the right foot, one at the base of the third toe and the other one at the level of the right ankle. The tissue body composition was analyzed using hand to foot resistance and reactance determined by resistance and reactance from an alternating current $(50 \mathrm{kHz})$.

\section{Body composition by dual-X-ray absorptiometry}

Dual-X-ray absorptiometry (DXA) measures were carried out using a bone densitometer (Lunar, GE Medical Systems) in the Hetmanska Medical Centre in Poznań. Bone mineral, lean and fat soft tissue were quantified by their absorption of photons emitted at two energy levels. Total and regional scans were taken. Two regions were considered of interest in this study: android fat distribution and gynoid fat distribution, both considered as the percentage of total body fat. Then the ratio of android to gynoid percentage (A/G ratio) was calculated.

\section{Statistical analysis}

Statistical analysis was carried out using STATISTICA software for Windows (version 6). Results are shown as mean \pm SEM. Continuous variables were assessed for normal distribution using the Shapiro-Wilk test. Logarithmic transformation was used to normalize non-normally distributed dependent variables. The associations between variables were calculated as the Pearson coefficient of correlation. A value of $P<0.05$ was regarded as a significant difference. All calculations and statistics were performed with STATISTICA for Windows version 6.0.

\section{Results}

In examined women we observed a normal metabolic profile - all lipoproteins were within the normal range. OGTT also proved to be normal. Only all insulin measurements were high: both insulin concentration in the fasting state and after a glucose load were increased; the HOMA-R index was also found to be high and indicative for the insulin resistance state.

From 50 women who entered the study, 33 were classified as insulin resistant (IR subgroup) and 17 as non-insulin resistant (non-IR subgroup), on the basis of their HOMA indices $(\geq 2.5$ or $<2.5$ ). IR women presented higher waist circumference, $A / G$ ratio measured by DXA, and a trend to higher WHR. Percentage of body fat measured by bioimpedance did not differ between the two subgroups.

The IR subgroup demonstrated a disadvantageous metabolic profile: elevated fasting triglycerides and glucose and higher insulin response in OGTT. In the non-IR subgroup we observed a 2.5 -fold increase of insulin 2 hours after the glucose load, while the IR group exhibited its 4.1-fold elevation.

We found significant positive correlations between measures of visceral fat and insulin: waist circumference and HOMA $(r=0.41)$, WHR and HOMA $(r=0.39)$, the percentage of android fat measured by DXA and HOMA $(r=0.40), A / G$ ratio and insulin $2(r=0.50)$, and A/G ratio and HOMA index $(r=0.51)$. Correlations with percentage of fat mass measured by bioimpedance and insulin were weaker; with insulin $0 r=0.29$ and with HOMA $r=0.31$.

From different insulin measurements (HOMA, insulin 0 , insulin 2, AUC insulin) HOMA index turned out to have the strongest correlations with the metabolic parameters triglycerides $(r=0.42)$ and glucose $0(r=0.34)$.

Finally we found a strong positive correlation between $W H R$ and A/R ratio measured by DXA (0.71, $p=0.0004)$ and only a trend with percentage of android fat measured by DXA (0.38).

\section{Discussion}

From 50 women who entered the study, 33 were classified as insulin resistant (IR subgroup) and 17 as 
non-insulin resistant (non-IR subgroup), according to their HOMA-R indices $(\geq 2.5$ or $<2.5)$. IR women presented higher waist circumference, $A / G$ ratio measured by DXA, and a trend to higher WHR. Percentage of body fat measured by bioimpedance did not differ between the two subgroups.

The IR subgroup demonstrated a disadvantageous metabolic profile: elevated fasting triglycerides and glucose and higher insulin response in OGTT (but most in the normal range). As in the non-IR subgroup we observed a 2.5-fold increase of insulin 2 hours after the glucose load, while the IR group revealed its 4.1-fold elevation.

Clinical studies have determined a high correlation between insulin resistance and the amount of intraabdominal fat [14]. Visceral fat tissue has metabolic properties making it significantly different from subcutaneous fat tissue, and responsible for its important role in the induction of insulin resistance. First, visceral, and especially intra-abdominal, fat tissue is characterized by resistance to the physiologic lipolytic action of insulin [15]. On the other hand, this kind of fat displays through its b3-adrenergic receptors high sensitivity to catecholamines, which promote lipolysis [16]. Hence, visceral obesity is accompanied by increased production of free fatty acids, which are transported through the portal circulation to the liver and further to peripheral tissues, especially the skeletal muscle. As a result, insulin resistance of peripheral tissues increases [16]. Other products originating from fatty tissue, such as leptin, tumor necrosis factor- $\alpha$ (TNF- $\alpha$ ), interleukin-2, $-6,-8$, resistin, and adiponectin, are also transported to distant tissues, inducing insulin resistance and being responsible for metabolic abnormalities.

Tab. I. Characteristics of examined group - anthropometry

\begin{tabular}{lcc}
\hline Factors & Mean & SEM \\
\hline Age (years) & 34.2 & 6.5 \\
\hline Height $(\mathrm{cm})$ & 164.9 & 8.9 \\
\hline Weight $(\mathrm{kg})$ & 104.0 & 22.0 \\
\hline BMI $\left(\mathrm{kg} / \mathrm{m}^{2}\right)$ & 39.0 & 9.7 \\
\hline Waist circumference $(\mathrm{cm})$ & 105.4 & 13.7 \\
\hline Hip circumference $(\mathrm{cm})$ & 125.5 & 13.7 \\
\hline WHR & 0.84 & 0.11 \\
\hline $\begin{array}{l}\text { Percentage fat body mass } \\
\text { bioimpedance (\%) }\end{array}$ & 47.3 & 11.7 \\
\hline $\begin{array}{l}\text { Percentage lean body mass } \\
\text { bioimpedance (\%) }\end{array}$ & 56.7 & 10.2 \\
\hline Percentage fat mass DXA (\%) & 52.6 & 11.0 \\
\hline Android fat distribution DXA (\%) & 51.9 & 10.2 \\
\hline Gynoid fat distribution DXA (\%) & 49.2 & 10.3 \\
\hline A/G ratio DXA & 1.03 & 0.07 \\
\hline $\begin{array}{l}\text { BMI - body mass index, WHR - waist-to-hip ratio, A/G ratio - android/ } \\
\text { gynoid ratio }\end{array}$ & \\
\hline
\end{tabular}

Tab. II. Characteristics of examined group - metabolic abnormalities

\begin{tabular}{lcc}
\hline Factors & Mean & SEM \\
\hline Total cholesterol $(\mathrm{mg} / \mathrm{dL})$ & 198.2 & 32.6 \\
\hline LDL $(\mathrm{mg} / \mathrm{dL})$ & 121.7 & 32.8 \\
\hline HDL $(\mathrm{mg} / \mathrm{dL})$ & 48.9 & 14.6 \\
\hline Triglycerides $(\mathrm{mg} / \mathrm{dL})$ & 130.6 & 50.0 \\
\hline Glucose 0 $(\mathrm{mg} / \mathrm{dL})$ & 92.3 & 11.3 \\
\hline Glucose 2 $(\mathrm{mg} / \mathrm{dL})$ & 119.8 & 22.5 \\
\hline AUC glucose $(\mathrm{mg} / \mathrm{dL} \times 120 \mathrm{~min})$ & 212.4 & 28.4 \\
\hline Insulin O $(\mathrm{nmol} / \mathrm{L})$ & 22.8 & 52.6 \\
\hline Insulin 2 $(\mathrm{nmol} / \mathrm{L})$ & 84.3 & 63.9 \\
\hline AUC Insulin $(\mathrm{nmol} / \mathrm{l} \times 120 \mathrm{~min})$ & 107.1 & 86.9 \\
\hline HOMA-R & 4.32 & 3.11 \\
\hline
\end{tabular}

$\mathrm{LDL}$ - low-density lipoprotein, $\mathrm{HDL}$ - high-density lipoprotein, AUC - area under the curve, HOMA-IR - homeostatic model assessment insulin resistance
In this study we were trying to determine whether any of the examined parameters (anthropometric or metabolic) would be helpful in indicating a target group for obesity pharmacological treatment with metformin. In the examined women we observed a normal metabolic profile: all lipoproteins were within the normal range. OGTT was also found to be normal. Only all insulin measurements were elevated: both insulin concentrations in the fasting state and after a glucose load were increased; HOMA-R indices also emerged to be high and indicative for the insulin resistance state. From different insulin measurements (HOMA, insulin 0, insulin 2, AUC insulin) HOMA index turned out to have the strongest correlations with the metabolic parameters triglycerides and glucose 0 . But unfortunately insulin is not a part of a standard approach in obesity in the primary care setting.

Moreover, in the whole examined group we found significant positive correlations between measures of visceral fat and insulin: waist circumference and HOMA, WHR and HOMA, the percentage of android fat measured by DXA and HOMA, A/G ratio and insulin 2, and $A / G$ ratio and HOMA index. In conclusion, in our study measures of DXA visceral fat determined by android fat percentage and $A / G$ ratio were the strongest determinants of insulin resistance.

DXA is a very potent method assessing not only total body fat and percentage of body fat mass, but also some specific measurements, such as visceral adipose tissue (VAT) and A/G ratio. Therefore it is a useful tool in prediction of cardiovascular risk and mortality [17, 18]. Based on DXA, with specific measurements such as changes in fat and lean body mass or changes in 
Tab. III. Characteristics of examined group - metabolic abnormalities in two subgroups IR and non-IR

\begin{tabular}{|c|c|c|c|c|c|}
\hline \multirow{2}{*}{ Factors } & \multicolumn{2}{|c|}{ IR subgroup, $n=33$} & \multicolumn{2}{|c|}{ Non-IR subgroup, $n=17$} & \multirow[t]{2}{*}{$P$} \\
\hline & Mean & SEM & Mean & SEM & \\
\hline HOMA-IR & 5.71 & 2.89 & 2.04 & 3.77 & $<0.05$ \\
\hline Waist circumference $(\mathrm{cm})$ & 110.9 & 13.1 & 98.3 & 12.6 & $<0.05$ \\
\hline Hip circumference $(\mathrm{cm})$ & 125.5 & 13.7 & 127.0 & 14.8 & NS \\
\hline WHR & 0.84 & 0.11 & 0.90 & 0.13 & NS, 0.08 \\
\hline Percentage fat body mass bioimpedance (\%) & 48.2 & 11.1 & 46.7 & 13.7 & NS \\
\hline Percentage lean body mass bioimpedance (\%) & 55.6 & 10.3 & 57.9 & 11.5 & NS \\
\hline Percentage fat mass DXA (\%) & 53.7 & 11.3 & 52.6 & 10.8 & NS \\
\hline Android fat DXA (\%) & 54.0 & 10.1 & 51.7 & 11.3 & NS \\
\hline Gynoid fat DXA (\%) & 50.1 & 9.07 & 48.2 & 12.4 & NS \\
\hline $\mathrm{A} / \mathrm{G}$ ratio $\mathrm{DXA}$ & 1.15 & 0.06 & 0.98 & 0.04 & $<0.05$ \\
\hline Total cholesterol (mg/dL) & 203.6 & 30.4 & 189.5 & 30.5 & NS \\
\hline LDL (mg/dL) & 116.5 & 29.7 & 125.1 & 26.9 & NS \\
\hline $\mathrm{HDL}(\mathrm{mg} / \mathrm{dL})$ & 45.2 & 14.6 & 51.7 & 14.4 & NS \\
\hline Triglycerides (mg/dL) & 147.0 & 46.1 & 122.9 & 52.0 & $<0.05$ \\
\hline Glucose 0 (mg/dL) & 98.1 & 11.0 & 84.2 & 14.7 & NS \\
\hline Glucose 2 (mg/dL) & 144.6 & 21.8 & 117.0 & 21.9 & $<0.05$ \\
\hline AUC glucose $(\mathrm{mg} / \mathrm{dL} \times 120 \mathrm{~min})$ & 242.8 & 28.4 & 201.3 & 23.9 & $<0.05$ \\
\hline Insulin 0 (nmol/L) & 27.3 & 56.1 & 15.0 & 47.0 & NS \\
\hline Insulin 2 (nmol/L) & 112.0 & 69.2 & 51.3 & 64.1 & $<0.05$ \\
\hline AUC insulin (nmol/l × $120 \mathrm{~min})$ & 139.2 & 86.3 & 68.4 & 77.4 & $<0.05$ \\
\hline
\end{tabular}

$\mathrm{BMI}$ - body mass index, WHR - waist-to-hip ratio, A/G ratio - android/gynoid ratio, LDL - low-density lipoprotein, HDL - high-density lipoprotein, AUC area under the curve, HOMA-IR - homeostatic model assessment insulin resistance

visceral fat we may assess more precisely the benefits of a weight reduction program and its elements, for example increased physical activity or a high-protein diet [19]. It is documented that additional DXA measurements, such as A/G fat ratio, similarly to WHR, correlate with unfavorable lipid profile and insulin resistance [20, 21]. In comparison to magnetic resonance imaging (MRI) DXA has a shorter scan time and lower radiation $[22,23]$.

Therefore the official positions of the International Society for Clinical Densitometry: Body Composition Analysis Reporting in 2013 stated: "The use of DXA adiposity measures (percent fat mass or fat mass index) may be useful in risk stratifying in patients for cardiometabolic outcomes" [24]. But unfortunately "Specific thresholds to define obesity have not been established" [22]. In the experts' opinion as BMI may be a part of a routine panel used for assessment of obese patients, it misclassifies a large segment of the population. The overclassification is associated with high musculature, more frequent in men. On the other hand, in women we often observe low musculature and high fat mass, leading to "normal weight obesity" [25]. As specific cutoffs to define obesity by the DXA method have not been established, some experts propose values of $20-25 \%$ of percent body fat in men, $30-35 \%$ in women, and up to $45 \%$ in the elderly [26], while others from the American Society for Bariatric Physicians advocate using values $>25 \%$ in men, and $>30 \%$ in women [22]. Taking into account the above considerations, in the absence of clear guidelines, although the DXA technique is widely used in clinical studies concerning obesity, it has still not become part of the routine examination of obese patients.

This study was designed in order to verify indications for metformin use in obese, non-diabetic subjects based on biochemical and anthropometric parameters including android fat content assessed by DXA. As there are no established indications for metformin treatment in obesity, we have demonstrated that women with high android fat content associated with elevated insulin resistance would benefit from metformin. Finally, as we found a strong positive correlation between WHR 
and $A / R$ ratio measured by DXA, we conclude that WHR might be a simple method for determining the indications for metformin treatment in obese premenopausal women. This was confirmed by other workers. For instance, in Indian men anthropometric measurements are good predictors of glycemia and insulin resistance. DXA makes only a small addition to the prediction [27].

The efficacy of metformin for the treatment of obesity has been evaluated in a few clinical trials with inconclusive results, but lately we can observe a rising number of convincing facts indicating its advantageous action in simple obesity. For instance, in low birth weight girls, metformin therapy was followed by a favorable adipokine profile and by a reduction of total, visceral, and hepatic adiposity beyond puberty [28]. Metformin is an effective drug to reduce weight in an outpatient setting in insulin sensitive and insulin resistant overweight and obese patients [29]. A meta-analysis of 14 trials, two on women with polycystic ovary syndrome (PCOS), demonstrated that metformin treatment reduced BMI significantly compared with placebo. The authors emphasize the greater effect of high-dose metformin and longer duration of therapy [30].

Limitations: This is a pilot study. In future works we plan to determine the effects of pharmacological intervention (including metformin) in obese premenopausal women.

\section{Conclusions}

In our study $67 \%$ of premenopausal obese women turned out to be insulin resistant on the basis of their HOMA-R indices. DXA measures of visceral fat determined by android fat percentage and android/gynoid ratio were the strongest determinants of insulin resistance. Waist-to-hip ratio might be a simple method for determining the indications for metformin treatment.

\section{Acknowledgements}

The research was supported in part by a grant from the Ministry of Science and Higher Education, Poland (NN 404127438).

\section{Disclosure}

The authors report no conflict of interest.

\section{References}

1. Friedman JM. Obesity in the new millennium. Nature 2000; 404: 632634.

2. Greenfield JR, Campbell LV. Insulin resistance and obesity. Clin Dermatol 2004; 22: 289-295

3. Ferrannini E, Balkau B, Coppack SW, et al. Insulin resistance, insulin response, and obesity as indicators of metabolic risk. J Clin Endocrinol Metab 2007; 92: 2885-2892.
4. Suliburska J. A six-week diet high in fat, fructose, and salt disturbs lipid and mineral status, in rats. Acta Sci Pol Technol Aliment 2013; 12: 195-202.

5. Bloomgarden ZT. Insulin resistance: current concepts. Clin Ther 1998; 20: 216-231.

6. Clinical guidelines in diabetes. Polish Diabetes Society 2013. Clinical Diabetology 2013, 2 Suppl. A.

7. American Diabetes Association. Standards of Medical Care in Diabetes - 2013. Diabetes Care 2013; 36: S11-S66.

8. Clinical guidelines on the identification, evaluation, and treatment of overweight and obesity in adults: executive summary. Expert Panel on the Identification, Evaluation, and Treatment of Overweight in Adults. Am J Clin Nutr 1998; 68: 899-917.

9. Flegal KM, Shepherd JA, Looker AC, et al. Comparison of percentage body fat, body mass index, waist circumference, and waist-stature ratio in adults. Am J Clin Nutr 2009; 89: 500-508.

10. Tsigos C, Hainer V, Basdevant A, et al.; Obesity Management Task Force of the European Association for the Study of Obesity. Management of obesity in adults: European clinical practice guidelines. Obes Facts 2008; 1: 106-116.

11. Matsuda M. Measuring and estimating insulin resistance in clinical and research settings. Nutr Metab Cardiovasc Dis 2010; 20: 79-86.

12. Belfiore $F$. Insulin resistance in obesity: metabolic mechanisms and measurement methods. Mol Genet Metab 1998; 65: 121-128.

13. Kawada T. Preliminary report: homeostasis model assessment of insulin resistance, an indicator of insulin resistance, is strongly related to serum insulin: practical data presentation and the mathematical basis. Metab Clin Exp 2010; 59: 1044-1046.

14. Banerji M, Chaiken R, Gordon D, et al. Does intraabdominal adipose tissue in black men determine whether NIDDM is insulin-resistant or insulin-sensitive? Diabetes 1995; 44: 141-146.

15. Lonqvist F, Thorne A, Nilsell K, et al. A pathogenetic role of visceral fat beta 3-adrenoreceptors in obesity. J Clin Invest 1995; 95: 1109-1116.

16. Krief S, Lonqvist F, Raimbault $S$, et al. Tissue distribution of beta-3-adrenergic receptor mRNA in man. J Clin Invest 1993; 91: 344-349.

17. Katzmarzyk PT, Greenway FL, Heymsfield SB, et al. Clinical utility and reproducibility of visceral adipose tissue measurements derived from dual-energy X-ray absorptiometry in White and African American adults. Obesity (Silver Spring) 2013; 21: 2221-2224; doi: 10.1002/oby.20519.

18. Kuk JL, Katzmarzyk PT, Nichaman MZ, et al. Visceral fat is an independent predictor of all-cause mortality in men. Obesity (Silver Spring) 2006; 14: 336-341.

19. Josse AR, Atkinson SA, Tarnopolsky MA, et al. Increased consumption of dairy foods and protein during diet- and exercise-induced weight loss promotes fat mass loss and lean mass gain in overweight and obese premenopausal women. J Nutr 2011; 141: 1626-1634.

20. Niederauer CM, Binkley TL, Specker BL. Effect of truncal adiposity on plasma lipid and lipoprotein concentrations. J Nutr Health Aging 2006; 10: $154-160$.

21. Aucouturier J, Meyer M, Thivel D, et al. Effect of android to gynoid fat ratio on insulin resistance in obese youth. Arch Pediatr Adolesc Med 2009; 163: 826-831.

22. Petak S, Barbu CG, Yu EW, et al. The official positions of the international society for clinical densitometry: body composition analysis reporting. J Clin Densitom 2013; 16: 508-519.

23. Micklesfield LK, Goedecke JH, Punyanitya M, et al. Dual-energy X-ray performs as well as clinical computed tomography for the measurement of visceral fat. Obesity (Silver Spring) 2012; 20: 1109-1114.

24. Nelson TL, Bessesen DH, Marshall JA. Relationship of abdominal obesity measured by DXA and waist circumference with insulin sensitivity in Hispanic and non-Hispanic white individuals: the San Luis Valley Diabetes Study. Diabetes Metab Res Rev 2008; 24: 33-40.

25. Shah NR, Braverman ER. Measuring adiposity in patients: the utility of body mass index (BMI), percent body fat, and leptin. PLoS One 2012; 7: e33308.

26. Okorodudu DO, Jumean MF, Montori VM, et al. Diagnostic performance of body mass index to identify obesity as defined by body adiposity: a systematic review and meta-analysis. Int J Obes (Lond) 2010; 34: 791-799.

27. Ganpule-Rao A, Joglekar C, Patkar D, et al. Associations of trunk fat depots with insulin resistance, $\beta$ cell function and glycaemia - a multiple technique study. PLoS One 2013; 8: e75391. 
28. Ibáñez L, Lopez-Bermejo A, Diaz M, et al. Pubertal metformin therapy to reduce total, visceral, and hepatic adiposity. J Pediatr 2010; 156: 98-102.

29. Seifarth C, Schehler B, Schneider HJ. Effectiveness of metformin on weight loss in non-diabetic individuals with obesity. Exp Clin Endocrino Diabetes 2013; 121: 27-31.

30. Nieuwenhuis-Ruifrok AE, Kuchenbecker WK, Hoek A, et al. Insulin sensitizing drugs for weight loss in women of reproductive age who are overweight or obese: systematic review and meta-analysis. Hum Reprod Update 2009; 15: 57-68. 